\title{
Enunciación
}

http://revistas.udistrital.edu.co/ojs/index.php/enunc

DOI: http://dx.doi.org/10.14483/udistrital.jour.enunc.2016.1.a03

enunciación

Artículo de Reflexión

\section{Análisis crítico del discurso: un discurso ecológico que integra el componente social}

\author{
Critical discourse analysis: An ecological discourse that integrates the social \\ component
}

\author{
Sonia Amparo Guerrero Cabrera ${ }^{1}$
}

Para citar este artículo: Guerrero, S. (2016). Análisis crítico del discurso: un discurso ecológico que integra el componente social. Enunciación, 21(1), 45-60.

Recibido: 26-febrero-2016 / Aprobado: 20-abril-2016

Como tantas veces ha ocurrido en el desarrollo del pensamiento humano, lo que conduce a las revoluciones intelectuales es más bien un cambio de perspectiva que una información nueva.

Paul Davies (1983).

\section{Resumen}

El proyecto de investigación -finalizado- denominado "Reconstrucción analítica de un discurso ecológico (RADE)", tuvo como objetivo central el reconocimiento de la realidad ecológica de Colombia, en cuanto muchas de sus dimensiones y componentes permanecen desconocidos para la población de Colombia. De esta manera se recurrió a las publicaciones que en el diario La República hizo la directora del Instituto de Investigación de Recursos Biológicos Alexander von Humboldt -es decir, una experta en el tema-, durante el periodo 2013-2014, se trata de 43 artículos en total.

Esta investigación aborda la problemática ambiental desde un enfoque poco usual: el análisis del discurso, cuyos postulados centrales, enunciados por Van Dijk, se aplican en cuanto al nivel semántico y pragmático. Por efectos metodológicos, en este artículo se da cuenta de los hallazgos a nivel semántico, donde tras el análisis se evidencia la importancia del componente social en la comprensión de la situación ecológica del país, es decir que uno de los resultados más significativos de esta investigación se orienta a resaltar el papel protagónico de elementos como la cultura, las tradiciones, la lengua, la historia y demás fenómenos sociales a la hora de entender e intervenir en una problemática que involucra a toda la ciudadanía: la gestión ambiental integral.

Palabras clave: análisis del discurso, ecología, sociedad, semántica, Colombia.

\begin{abstract}
To contribute to the recognition of the ecological reality of Colombia, the project called Reconstruction Analytical of an Ecological Speech, which retakes the publications in the newspaper The Republic made by the director of the Alexander von Humboldt Biological Resources
\end{abstract}

1 Magíster en Hermenéutica Literaria. Docente Facultad de Educación, Universidad de San Buenaventura. Correo electrónico: sonia.guerrero@ usbmed.edu.co 
Research Institute, during 2013 - 2014, and are the object of this study: it is 43 articles in total. This research addresses environmental issues from an unusual approach: discourse analysis. The aim of this paper is to highlight the social component of the ecological situation in the country, constantly

\section{INTRODUCCIÓN}

Colombia es conocida como el país de la megadiversidad (Baptiste, 2013f), por la abundancia de sus recursos naturales, la gran variedad de flora, fauna, y cultura, como expresión de las relaciones que el ser humano establece con su entorno. De esto se deduce que el manejo de biodiversidad es fundamental para el bienestar humano y el de todas las especies que la conforman, lo cual posibilita la preservación de la vida. Por consiguiente, estudiar los diferentes factores que integran dicho bienestar es el camino para implementar mejoras en la calidad de la vida humana y en el cuidado del medio ambiente, dimensiones que no pueden desligarse y que se influyen mutuamente. Es decir, hay una relación directa entre todas las construcciones culturales y sociales humanas y los procesos ecosistémicos; la comprensión de dichas dinámicas es un asunto importante para el progreso con carácter sostenible. El objetivo de la investigación RADE es, a través de la identificación y el análisis de los factores que integran la situación ecológica del país, reconocer los componentes que desde lo social influyen en el modo de vida de los colombianos.

Si se asume que la ecología es "la ciencia que estudia las interacciones de los organismos vivos y su ambiente" (Sutton, 2008, p. 25), es más fácil discernir que los procesos que ocurren en la naturaleza no pueden tomarse de forma aislada, los organismos vivos interactúan entre sí y con los componentes químicos y físicos inanimados, de esa manera se va componiendo un sistema en el que actúan diferentes factores, esto es lo que se denomina ecosistema.

Su funcionamiento es como un sistema abierto de flujo de energía, donde hay constantes intercambios emphasized as a relevant aspect in the articles of the autor, as shown in the reconstruction of the semantic level.

Keywords: discourse analysis, ecology, society, semantics, Colombia.

que tienden a equilibrar las condiciones del mismo. Los límites de un ecosistema no son claros, debido a las intrincadas relaciones que los componen: es un sistema esencialmente dinámico, abierto y vulnerable.

La vulnerabilidad de los ecosistemas hace que cada intervención humana cause un impacto que no siempre se reconoce. El riesgo ecológico, o crisis ambiental, es famoso, pero la complejidad de las intrincadas relaciones que lo habitan pasan desapercibidas para la mayoría de la población, en especial la urbana; y teniendo en cuenta que es en la ciudad donde se toman las decisiones de gestión ambiental, dichas resoluciones no están completamente contextualizadas. Tomando como ejemplo el artículo de Baptiste (2013c) Las economías del páramo, resulta esclarecedor el hecho de que es posible usar los recursos naturales -en este caso el agua-, y hacer de ello una labor sostenible. El tema central del artículo es la "Conservación y explotación del páramo de acuerdo con las características ecosistémicas del territorio", lo que implica cuatro factores principales: proteger los servicios ecosistémicos; aprovechar las características socioculturales de los pobladores; reconocer los límites de la división territorial; y realizar una evaluación del impacto ambiental. Se evidencia así que la gestión ambiental debe trabajar en varios frentes para sumir el manejo de la biodiversidad. El develamiento de estos procesos en términos claros, sencillos y accesibles se convierte en una contribución muy valiosa en el proceso de innovación y evolución humana, ya que el ser humano, a través de los conocimientos que acumula y por el estudio de fenómenos -con base en esos conocimientos-, es capaz de transformar su entorno. Además, de permitirle tomar decisiones 
que integren las diferentes variables (ambientales y sociales) que se ponen en juego en toda intervención humana en el mundo.

De esta forma, para poner de manifiesto los factores que se acaban de mencionar se recurrió al análisis crítico del discurso (ACD), planteado por Teun A. van Dijk, que como primer paso investigativo propone unas categorías específicas de definición y reconocimiento de un fenómeno como discurso para pasar a realizar el análisis. El discurso es:

[...] un acontecimiento comunicativo específico. Ese evento comunicativo es en sí mismo bastante complejo, y al menos involucra a una cantidad de actores sociales, esencialmente en los roles de hablante/escribiente y oyente/lector (pero también en otros roles, como observador o escucha), que intervienen en un acto comunicativo, en una situación específica (tiempo, lugar, circunstancias) y determinado por otras características del contexto (Londoño, 2011, p. 103).

Todos estos elementos se identifican en las publicaciones de Baptiste, la autora de los artículos a analizar, avalando así teóricamente su análisis. Teniendo en cuenta que la misión del Instituto de Investigación de Recursos Biológicos Alexander von Humboldt es promover, coordinar y realizar investigación que contribuya al conocimiento, la conservación y el uso sostenible de la biodiversidad, como un factor de desarrollo y bienestar de la población colombiana, es compromiso de la directora brindar a esa población los datos, herramientas y conclusiones que surgen a la hora de convivir con la naturaleza. El discurso de divulgación ecológica de Baptiste se legitima por su autoridad en el tema, es un acto de comunicación pública ubicado geográfica y culturalmente. Sus artículos constituyen un escenario propicio para investigar a través del ACD, el tema de la ecología colombiana, centrada en la comprensión de los riesgos que existen actualmente, y por consiguiente en las fortalezas.

La intención de este escrito es dar a conocer los hallazgos que a nivel de análisis semántico indican no solo los riegos ecológicos que el país afronta, sino la influencia de los fenómenos sociales en las causas, consecuencias y soluciones de dicha situación. El componente social que se nombra hace referencia a todos aquellos procesos culturales, históricos, políticos, económicos: todo lo que construye el ser humano para relacionarse con la realidad. Se espera que la comunicación de esta información contribuya a la formación de un criterio más amplio en la ciudadanía a la hora de pensar el territorio, la biodiversidad, la urbe, lo rural y otros, asociados a la convivencia del ser humano en la sociedad.

\section{METODOLOGÍA: BASADA EN LOS POSTULADOS TEÓRICOS DE VAN DIJK SOBRE EL ANÁLISIS CRÍTICO DEL DISCURSO (ACD)}

La teoría propuesta por Van Dijk es bastante amplia, por lo que este trabajo se centra en las nociones fundamentales del nivel semántico, y en el objetivo de la investigación, que se enuncia así: identificar a través del análisis crítico del discurso las características del discurso de la directora del Instituto de Investigación de Recursos Biológicos Alexander von Humboldt, que permiten la comprensión de los principales riesgos ecológicos de Colombia.

La intención de comenzar con este nivel radica en la importancia de establecer las proposiciones que presenta el discurso, los temas que los componen y determinar la coherencia de los mismos -COherencia global con las macrorreglas y coherencia local con las microestructuras de significado-, todo esto para que las conclusiones y hallazgos tengan su cuna en un discurso del que se reconocen sus partes y lógica de construcción. Por su parte las microestructuras de significado se analizan para acabar de consolidar los componentes esenciales de los artículos, como forma exhaustiva de abarcar los conceptos, ideas y propuestas de los mismos. Por medio del estudio de estos apartados se pudo establecer el objetivo de la autora con sus textos, que se puede resumir en la ampliación de la visión de la situación ecológica desde el cuidado acostumbrado del medio ambiente -que falla hoy en día-, para 
avanzar hacia la gestión ambiental integral, que involucra al ser humano no como un agente externo que controla y dirige, sino como elemento esencial del ecosistema, donde toda acción y creación tienen su origen y su fin en la naturaleza.

En primer lugar, se retoman los postulados del ACD sobre el uso de la lengua como evento de comunicación e interacción en diferentes contextos cognitivos, sociales, políticos, históricos y culturales; y su enfoque comprometido en explicar y develar las manifestaciones del poder, ya que así promueve cambios en las condiciones sociales. Lo que deviene en que los investigadores o analistas críticos deben poseer una conciencia o posición explícita y activa de su papel en la sociedad y ante una problemática específica.

Así, la elaboración de teoría, la descripción y la explicación sobre el discurso están situadas sociopolíticamente, buscan generar un cambio en el entendimiento de las relaciones entre discurso y sociedad, y mediante la explicación combaten la reproducción del abuso de poder y la desigualdad. "Un análisis crítico tiene como objetivo fundamental evidenciar, a través del análisis del discurso, problemas sociales y políticos" (Van Dijk, 1994, p. 6).

Sus métodos son cualitativos de descripción detallada de estructuras y estrategias a nivel sintáctico (estructuras formales de las oraciones), semántico (estructuras de sentido y referencia), pragmático (actos del habla), de interacción y conversación, de procesos y representaciones mentales, y las relaciones de todas esas estructuras con el contexto en el que ocurren. Todo esto con una aproximación práctica que no solo describa sino que explique el uso del lenguaje y del discurso.

De todo ello deviene que el ACD es multidisciplinario, permite examinar los diferentes componentes lingüísticos de un discurso y generar múltiples hallazgos, para los objetivos de esta investigación -comprender los principales riesgos ecológicos de Colombia-, la atención se centra en la macroestructura, compuesta por la semántica y la pragmática. En este escrito se aborda únicamente el nivel semántico para optimizar el impacto de los hallazgos.

La semántica habilita entre otras cosas examinar el sentido de un discurso, identificar su coherencia, lo que la convierte como se dijo en el punto de partida de esta investigación. La estructura semántica de un discurso hace explícito el tema, tal estructura no se expresa en oraciones individuales sino en secuencias completas de las mismas, en lo que se denomina macroestructuras semánticas (Van Dijk, 1998, p. 43) y permite conocer la coherencia global de un discurso: establecer su tema. Entonces, el sentido global de un discurso se deriva de la secuencia proposicional que subyace en él. Las proposiciones son los enunciados en los que se expresan los temas o asuntos de un discurso, y como forman parte de la macroestructura pasan a denominarse macroproposiciones (Van Dijk, 1998, p. 46).

El ACD se sirve del procedimiento de aplicación de macrorreglas para identificar la coherencia global; estas se usan posteriormente para determinar las proposiciones y tienen por función reducir, organizar y transformar la información semántica, estas son: supresión, generalización y construcción (Van Dijk, 1998, p. 49). La supresión elimina toda proposición que no sea presuposición de las subsiguientes proposiciones de la secuencia. La generalización retoma la nueva secuencia de proposiciones, y la sustituye por la original. En la construcción se fabrica una proposición que reúna y contenga el mismo evento denotado por la totalidad de la secuencia de proposiciones.

El principal aporte de las macrorreglas es el establecimiento del tema de un discurso, que en este ejercicio ilustra la importancia del componente social en el tema ambiental.

En el análisis crítico del discurso el nivel semántico permite además establecer la coherencia local del texto que posibilita evaluar las formas con que este mantiene las relaciones semánticas entres las proposiciones expresadas por oraciones o secuencias de oraciones. Es decir que su análisis permite, a partir de unas categorías específicas denominadas microestructuras de significado (Van Dijk, 1984, p. 
213), establecer las conexiones internas del texto. Dichas categorías son:

- Implicaturas: Son las nociones que tienen o manejan las personas sobre un tema; en un discurso no siempre se hace explicita la enunciación de todos los elementos. "Una característica bien conocida de la oración y la semántica del discurso consiste en que los significados no se expresan siempre de manera explícita, sino que suelen estar implicados semánticamente, o mediatizados por otras expresiones explícitas y sus significados" (Van Dijk, 2008, p. 234).

- Presuposición: Es una implicación semántica.

En términos formales, una proposición $q$ se presupone en $p$, si se implica tanto de $p$ como de no- $p$. En términos informales, cualquier proposición cuya verdad sea aceptada por el hablante se convierte en presuposición. Lingüísticamente, las presuposiciones son proposiciones verdaderas no asertivas que son significativas para la estructura de las oraciones. Como en el caso de las implicaciones, las presuposiciones permiten a los hablantes o escritores criticar sin afirmarlas (Van Dijk, 2008, p. 240).

Una presuposición es una proposición que permanece invariable en la negación y en la interrogación. Para determinarla se recurre a negar e interrogar para que la proposición resultante después de estos procesos, se constituya como el contenido implícito de una oración. Una presuposición "se desprende necesariamente del significado de las palabras enunciadas y de ella depende que una oración pueda someterse a la prueba veritativa" (Calsamiglia, 1999, p. 190), dicha prueba es la que se mencionó: someter al enunciado a la negación y pregunta del mismo.

- Repetición: Significa que en el desarrollo proposicional el autor de un texto repite el mismo término varias veces, no hay variación léxica. Puede obedecer a una lógica concreta, de refuerzo o introducción de un concepto.

- Sinonimia: Por razones muy variadas, como estilísticas o pedagógicas, un autor recurre ya sea para el enriquecimiento del vocabulario o para la ampliación de un concepto, entre otros, al uso de un término que reemplaza a otro como una variación, un término con una significación semejante que tenga una clara relación simétrica (Martínez, 1997, p. 45) con el anterior.

- Superordenación: Es un término que expresa un concepto clave, abarca la significación global del tema principal. Está unido al significado de otros términos importantes dentro del discurso, y la asociación que se establece entre ellos se realiza por medio de una relación de inclusión (Martínez, 1997, p. 46).

- Comparación: Se usa para atenuar o resaltar elementos y conceptos importantes en el discurso, generalmente se usa la palabra "como".

- Formas de tratamiento: La selección de específicas formas de nombrar y las formas de tratamiento de grupos y conceptos a los que recurre un autor, marcan la relación que establece quien nombra con lo nombrado (autor/discurso).

Hacia la parte final de este escrito se describen los resultados de estas categorías analíticas.

\section{RESULTADOS Y CONCLUSIONES}

Desde el ACD se puede decir que los artículos objeto de la investigación tienen todos una serie de proposiciones bastante precisas, ya que después de la aplicación de las macrorreglas, la supresión indica que un alto porcentaje de proposiciones permanecen; y además cada uno contiene un tema definido, es decir que la coherencia global es apropiada. Esto se logra en la medida en que se 
jerarquizan las ideas de forma que se presenta el asunto principal y los postulados que lo soportan. Aunque algunos temas son recurrentes, explican con cada aparición una nueva dimensión en la que inciden. Se establecen así entre ellos -los temas- relaciones de dependencia, causa y efecto, necesidades, y de contexto. En la tabla 1 aparecen los datos principales que se obtuvieron con el uso de las macrorreglas.

Los artículos se orientan a la caracterización de la gestión ambiental como un proceso importante para afrontar la crisis. Desde el primer artículo "Punto de partida" se menciona que dicha gestión se cimenta en la biodiversidad del país; en "El lugar de la biodiversidad en la paz", se resalta la influencia de dicha biodiversidad con sus características sociales y ecológicas para lograr una convivencia saludable y sostenible. En "La lora ambiental" se plantea al lector la necesidad de transformar las políticas ambientales actuales de forma que se movilicen los recursos naturales y los humanos; igual ocurre en "OCDE verde" que expresa la necesidad de una planificación sectorial del desarrollo, todo con leyes contextualizadas, es decir acordes a las necesidades del medio y de los pobladores.

Cuando se habla de la gobernabilidad del territorio que alude directamente a la gestión ambiental del Estado, los artículos mencionan la gran importancia del reconocimiento de las transiciones socioecológicas que están ocurriendo, que involucran procesos biológicos y culturales, como bien lo indica el artículo "Transiciones ecológicas y sociales en Santurbán", esto significa replantear todas las relaciones productivas y reproductivas entre las personas y con la biodiversidad, para lograr una reinterpretación del contexto territorial: habitar de forma sostenible el país.

En "La interfaz ciencia-política" se explica la necesaria creación de instituciones que medien en la relación de la ciencia con la política para integrarlas en la gestión ambiental; es decir, que científicos y políticos trabajen juntos, y así las prácticas y leyes ambientales generen beneficios en todos los contextos; en"El efecto silo" se reanuda el tema para agregar que el trabajo de las instituciones encargadas de la gestión ambiental debe ir en consonancia con las nuevas circunstancias biológicas y socioeconómicas que se presentan actualmente en el país. Para ampliar este tema, el artículo "Resiliencia", aborda dicho concepto como la capacidad adaptativa para afrontar la crisis actual, y se indica cómo la resiliencia se compone no solo de lo ecológico sino de lo social. Apoya esta noción el artículo "Ecología profética" que orienta sobre reconocimiento de los límites y alcances de las predicciones ecológicas en el mantenimiento de la resiliencia, explica que una buena investigación define y precisa factores y niveles de riesgo ante las posibles decisiones de intervención, ya que los cambios son constantes, por tanto, la planificación debe fortalecer la capacidad adaptativa del país, para asumir nuevas formas de pensar el mundo, de habitarlo. De estos elementos ecológicos deviene un concepto fundamental para la gestión ambiental: la innovación.

Para innovar ecológicamente, el artículo "Restaurando" asume como una parte primordial de la gestión ambiental los procesos de restauración, que requieren innovaciones que incorporen diferentes conocimientos, perspectivas y tecnologías, para abarcar la complejidad de los ecosistemas. Esto se complementa con lo propuesto en "Innovación ambiental", que es un Ilamado a reconocer e integrar las capacidades creativas de todos los sectores involucrados en el manejo del ambiente. Como ejemplos más relevantes, concretos y reales de la posibilidad de innovar en la gestión ambiental están las agrupaciones de fuerzas sociales que en el artículo "Transiciones ecológicas y sociales en Santurbán" buscan proteger el páramo de esa región; el artículo "Biomímesis" trata sobre la disciplina del diseño industrial así denominada, que básicamente consiste en la imitación de la naturaleza, es decir implementar los principios de regulación y sostenimiento de ella para el diseño de modos de vida saludables y ecológicos. 
Tabla 1. Datos principales de la aplicación de macrorreglas

\begin{tabular}{|c|c|c|c|c|}
\hline Artículo & $\begin{array}{c}\# \\
\text { Proposiciones }\end{array}$ & $\begin{array}{l}\text { \# Proposiciones } \\
\text { que permanecen }\end{array}$ & $\begin{array}{l}\text { Porcentaje } \\
\text { que } \\
\text { permanece }\end{array}$ & Tema \\
\hline Punto de partida & 36 & 24 & $67 \%$ & $\begin{array}{l}\text { El manejo de la biodiversidad, teniendo en cuenta } \\
\text { los factores y componentes que la hacen comple- } \\
\text { ja: circunstancias sociales y ecológicas del país. }\end{array}$ \\
\hline La lora ambiental & 32 & 16 & $50 \%$ & $\begin{array}{l}\text { La política ambiental del Estado entorpece más } \\
\text { que beneficiar la apropiada gestión de la biodi- } \\
\text { versidad. }\end{array}$ \\
\hline $\begin{array}{l}\text { Las economías del } \\
\text { páramo }\end{array}$ & 28 & 18 & $64 \%$ & $\begin{array}{l}\text { Conservación y explotación del páramo de acuer- } \\
\text { do con las características ecosistémicas del terri- } \\
\text { torio. }\end{array}$ \\
\hline $\begin{array}{l}\text { Ayuno y } \\
\text { abstinencia }\end{array}$ & 31 & 15 & $48 \%$ & $\begin{array}{l}\text { Consumo insostenible de recursos como resulta- } \\
\text { do de la constante búsqueda del placer promovi- } \\
\text { da por el mercado }\end{array}$ \\
\hline $\begin{array}{l}\text { Información } \\
\text { ambiental 1, } 2 \text { y } 3\end{array}$ & 32 & 20 & $63 \%$ & $\begin{array}{l}\text { Regulaciones en el manejo de la información so- } \\
\text { bre medio ambiente, que debe ser permanente, } \\
\text { accesible, relevante, oportuna y oficial para ga- } \\
\text { rantizar decisiones coherentes. }\end{array}$ \\
\hline $\begin{array}{l}\text { El lugar de la } \\
\text { biodiversidad en } \\
\text { la paz }\end{array}$ & 23 & 16 & $70 \%$ & $\begin{array}{l}\text { La biodiversidad influye en un modelo de convi- } \\
\text { vencia saludable, por lo que su gestión debe ba- } \\
\text { sarse en las características sociales y ecológicas } \\
\text { del país. }\end{array}$ \\
\hline $\begin{array}{l}\text { Desplazamiento } \\
\text { ambiental }\end{array}$ & 30 & 16 & $53 \%$ & $\begin{array}{l}\text { Los aspectos sociales, ecológicos y tecnológicos } \\
\text { que influyen en el uso adecuado del territorio, } \\
\text { para generar políticas de seguridad poblacional y } \\
\text { ambiental. }\end{array}$ \\
\hline $\begin{array}{l}\text { Territorios } \\
\text { colectivos y } \\
\text { biocapacidad }\end{array}$ & 24 & 14 & $58 \%$ & $\begin{array}{l}\text { Mantenimiento de la biocapacidad territorial, } \\
\text { donde las formas de gobernanza de las minorías } \\
\text { étnicas son la opción más acertada para un país } \\
\text { megadiverso. }\end{array}$ \\
\hline $\begin{array}{l}\text { La gobernanza } \\
\text { de las ficciones } \\
\text { territoriales }\end{array}$ & 22 & 14 & $64 \%$ & $\begin{array}{l}\text { La gobernanza efectiva del territorio requiere el } \\
\text { reconocimiento de sus condiciones biológicas y } \\
\text { culturales, además de leyes claras y pertinentes de } \\
\text { protección de la biodiversidad. }\end{array}$ \\
\hline $\begin{array}{l}\text { Innovación am- } \\
\text { biental }\end{array}$ & 29 & 17 & $59 \%$ & $\begin{array}{l}\text { La innovación en la gestión ambiental como prio- } \\
\text { ridad para lograr afrontar la crisis, al reconocer } \\
\text { e integrar las capacidades creativas de todos los } \\
\text { sectores involucrados. }\end{array}$ \\
\hline $\begin{array}{l}\text { Reforma rural } \\
\text { integral y servicios } \\
\text { ecosistémicos }\end{array}$ & 32 & 22 & $69 \%$ & $\begin{array}{l}\text { La construcción de reformas rurales que sosteni- } \\
\text { blemente protejan los servicios ecosistémicos, los } \\
\text { modos de vida y la producción agropecuaria de } \\
\text { las comunidades campesinas. }\end{array}$ \\
\hline OCDE verde & 25 & 12 & $48 \%$ & $\begin{array}{l}\text { Rescate de la gestión ambiental que necesita una } \\
\text { planificación sectorial del desarrollo y políticas } \\
\text { ambientales certeras y contextualizadas. }\end{array}$ \\
\hline $\begin{array}{l}\text { Transiciones } \\
\text { ecológicas y } \\
\text { sociales en } \\
\text { Santurbán }\end{array}$ & 22 & 11 & $50 \%$ & $\begin{array}{l}\text { Reconocimiento y ejecución de las transiciones } \\
\text { socioecológicas necesarias para una adecuada } \\
\text { gobernabilidad del territorio que orienten la ges- } \\
\text { tión ambiental. }\end{array}$ \\
\hline
\end{tabular}


Continuación Tabla 1.

\begin{tabular}{|c|c|c|c|c|}
\hline Artículo & $\begin{array}{c}\# \\
\text { Proposiciones }\end{array}$ & $\begin{array}{l}\text { \# Proposiciones } \\
\text { que permanecen }\end{array}$ & $\begin{array}{l}\text { Porcentaje } \\
\text { que } \\
\text { permanece }\end{array}$ & Tema \\
\hline $\begin{array}{l}\text { Travesías, } \\
\text { transiciones }\end{array}$ & 25 & 17 & $68 \%$ & $\begin{array}{l}\text { El viaje como forma de entender y proteger mejor } \\
\text { el territorio, y crear así una planeación integral y } \\
\text { equilibrada del uso de este. }\end{array}$ \\
\hline $\begin{array}{l}\text { Enfermedades } \\
\text { ecosistémicas }\end{array}$ & 31 & 16 & $52 \%$ & $\begin{array}{l}\text { Las enfermedades ecológicas trasforman drástica- } \\
\text { mente la naturaleza, amenazando los modos de } \\
\text { vida actuales y futuros y evidencian nuestro gran } \\
\text { desconocimiento de sus alcances e impactos eco- } \\
\text { lógicos. }\end{array}$ \\
\hline $\begin{array}{l}\text { Prosperidad con } \\
\text { límites }\end{array}$ & 27 & 14 & $52 \%$ & $\begin{array}{l}\text { Noción del límite planetario que orienta sobre la } \\
\text { construcción urgente de planes de desarrollo que } \\
\text { no sobrepasen la biocapacidad del territorio. }\end{array}$ \\
\hline $\begin{array}{l}\text { Ecologías de } \\
\text { escala }\end{array}$ & 27 & 17 & $63 \%$ & $\begin{array}{l}\text { Producción agropecuaria ecológica que com- } \\
\text { prende las propiedades ecosistémicas del territo- } \\
\text { rio y las características culturales de sus habitan- } \\
\text { tes, a la hora de generar sostenibilidad. }\end{array}$ \\
\hline Ecología profética & 31 & 17 & $55 \%$ & $\begin{array}{l}\text { Reconocimiento de los límites y alcances de las } \\
\text { predicciones ecológicas en el mantenimiento de } \\
\text { la resiliencia (capacidad adaptativa). }\end{array}$ \\
\hline $\begin{array}{l}\text { Empresas de ser- } \\
\text { vicios } \\
\text { ¿ecosistémicos? }\end{array}$ & 29 & 12 & $41 \%$ & $\begin{array}{l}\text { Entender la inversión en el buen manejo del agua } \\
\text { como una ganancia para el bienestar social y am- } \\
\text { biental. }\end{array}$ \\
\hline Restaurando & 27 & 17 & $63 \%$ & $\begin{array}{l}\text { Innovaciones en los procesos de restauración } \\
\text { donde se incorporen diferentes conocimientos, } \\
\text { perspectivas y tecnologías, para abarcar la com- } \\
\text { plejidad de los ecosistemas. }\end{array}$ \\
\hline Nada se toca & 35 & 22 & $63 \%$ & $\begin{array}{l}\text { Posiciones extremas frente los temas ambientales } \\
\text { que cierran el paso a la gestión e innovación de } \\
\text { estos, y donde nadie asume responsabilidades. }\end{array}$ \\
\hline $\begin{array}{l}\text { La interfaz } \\
\text { ciencia-política }\end{array}$ & 27 & 18 & $67 \%$ & $\begin{array}{l}\text { La necesaria creación de instituciones que me- } \\
\text { dien en la relación de la ciencia con la política } \\
\text { para integrarlas en la gestión ambiental. }\end{array}$ \\
\hline $\begin{array}{l}\text { Pacto ¿agro... } \\
\text { ambiental? }\end{array}$ & 27 & 17 & $63 \%$ & $\begin{array}{l}\text { La cultura agraria como forma de vida rural que } \\
\text { respeta las características ecosistémicas del terri- } \\
\text { torio y a sus habitantes. }\end{array}$ \\
\hline $\begin{array}{l}\text { Cesar las guerras } \\
\text { contra el agua }\end{array}$ & 26 & 17 & $65 \%$ & $\begin{array}{l}\text { La gran discrepancia entre el modelo mental del } \\
\text { mundo de los colombianos y la realidad ecológi- } \\
\text { ca y social, en especial con el manejo del recurso } \\
\text { hídrico. }\end{array}$ \\
\hline $\begin{array}{l}\text { Sostenibilidad en } \\
\text { desarrollo }\end{array}$ & 23 & 12 & $52 \%$ & $\begin{array}{l}\text { Los límites planetarios de biocapacidad implican } \\
\text { la comprensión de procesos sociales y ecológicos } \\
\text { para el desarrollo de la sostenibilidad: la agrega- } \\
\text { ción de ciclos de vida naturales o artificiales am- } \\
\text { plía el umbral de supervivencia. }\end{array}$ \\
\hline Gentileza & 25 & 14 & $56 \%$ & $\begin{array}{l}\text { Las poco estudiadas transiciones ecológicas de } \\
\text { las que surge un nuevo socioecosistema, que es } \\
\text { una oportunidad para construir un mundo soste- } \\
\text { nible en lo ecológico y lo social. }\end{array}$ \\
\hline
\end{tabular}


Continuación Tabla 1.

\begin{tabular}{|c|c|c|c|c|}
\hline Artículo & $\begin{array}{c}\# \\
\text { Proposiciones }\end{array}$ & $\begin{array}{l}\text { \# Proposiciones } \\
\text { que permanecen }\end{array}$ & $\begin{array}{l}\text { Porcentaje } \\
\text { que } \\
\text { permanece }\end{array}$ & Tema \\
\hline El efecto silo & 23 & 14 & $61 \%$ & $\begin{array}{l}\text { Ante la fragmentación sectorial promovida por los } \\
\text { estados modernos, la gestión integral requiere un } \\
\text { gobierno vinculante, acorde a las circunstancias } \\
\text { biológicas y socioeconómicas del país. }\end{array}$ \\
\hline Comer borugo & 26 & 13 & $50 \%$ & $\begin{array}{l}\text { Para la gestión de la biodiversidad colombiana } \\
\text { son muy importantes las tradiciones culturales, } \\
\text { debido al uso y manejo de los recursos naturales } \\
\text { que, en muchos casos, representan alternativas de } \\
\text { sostenibilidad. }\end{array}$ \\
\hline $\begin{array}{l}\text { Experimentando } \\
\text { los límites }\end{array}$ & 32 & 19 & $59 \%$ & $\begin{array}{l}\text { Alternativas innovadoras de generación de ener- } \\
\text { gía en el territorio isleño colombiano ante el dete- } \\
\text { rioro de sus condiciones de biocapacidad. }\end{array}$ \\
\hline Plantas del pasado & 20 & 12 & $60 \%$ & $\begin{array}{l}\text { Importancia de la preservación y buen uso de la } \\
\text { vasta flora silvestre colombiana por parte de todos } \\
\text { los actores sociales, para la gestión de la biodi- } \\
\text { versidad. }\end{array}$ \\
\hline Tierra cansada & 27 & 15 & $56 \%$ & $\begin{array}{l}\text { Necesidad de una política agropecuaria que ga- } \\
\text { rantice la calidad ambiental y nutricional de los } \\
\text { ecosistemas alimentarios del país debido al alto } \\
\text { requerimiento de insumos químicos en la produc- } \\
\text { ción agropecuaria. }\end{array}$ \\
\hline $\begin{array}{l}\text { Ministerio de la } \\
\text { fauna }\end{array}$ & 23 & 9 & $39 \%$ & $\begin{array}{l}\text { Políticas ambientales con enfoque sistémico para } \\
\text { abordar los eventos climáticos y la crisis ecosis- } \\
\text { témica. }\end{array}$ \\
\hline Resiliencia & 25 & 14 & $56 \%$ & $\begin{array}{l}\text { Manejo de la resiliencia social y ecológica del } \\
\text { país para la creación de un modelo de desarrollo } \\
\text { sostenible, acorde al contexto cambiante. }\end{array}$ \\
\hline Pagamentos & 22 & 13 & $59 \%$ & $\begin{array}{l}\text { Importancia de las regulaciones estatales en el } \\
\text { pago por los servicios ecosistémicos, para man- } \\
\text { tener el flujo de recursos y esfuerzos que crean } \\
\text { sostenibilidad. }\end{array}$ \\
\hline IPBES & 25 & 14 & $56 \%$ & $\begin{array}{l}\text { Urgencia de acuerdos entre todos los países para } \\
\text { afrontar la crisis ambiental: reconocimiento de fa- } \\
\text { llas y fortalezas en la gestión de la biodiversidad. }\end{array}$ \\
\hline $\begin{array}{l}\text { Innovación } \\
\text { ambiental (2) }\end{array}$ & 30 & 16 & $53 \%$ & $\begin{array}{l}\text { Un modelo de innovación del manejo de la biodi- } \\
\text { versidad basado en las tendencias culturales para } \\
\text { construir nuevos modos de desarrollo. }\end{array}$ \\
\hline $\begin{array}{l}\text { El árbol de la } \\
\text { abundancia }\end{array}$ & 25 & 12 & $48 \%$ & $\begin{array}{l}\text { Comprensión de la importancia de la biodiversi- } \\
\text { dad tanto en lo biológico como en los procesos } \\
\text { de producción para construir nuevos modos de } \\
\text { vida, incluyentes y equitativos. }\end{array}$ \\
\hline $\begin{array}{l}\text { Lógicas } \\
\text { posconsumo }\end{array}$ & $28 /$ & 15 & $54 \%$ & $\begin{array}{l}\text { El rol de las empresas como parte fundamental de } \\
\text { la adaptación y del mantenimiento energético de } \\
\text { los ecosistemas y por tanto de la sostenibilidad. }\end{array}$ \\
\hline $\begin{array}{l}\text { La fragilidad } \\
\text { ambiental de la } \\
\text { cultura }\end{array}$ & 24 & 11 & $46 \%$ & $\begin{array}{l}\text { Necesidad de una educación contextualizada a } \\
\text { las características ecológicas y sociales de cada } \\
\text { país, donde se aproveche la cultura en la gestión } \\
\text { ambiental. }\end{array}$ \\
\hline
\end{tabular}


Continuación Tabla 1.

\begin{tabular}{|c|c|c|c|c|}
\hline Artículo & $\begin{array}{c}\# \\
\text { Proposiciones }\end{array}$ & $\begin{array}{l}\text { \# Proposiciones } \\
\text { que permanecen }\end{array}$ & $\begin{array}{l}\text { Porcentaje } \\
\text { que } \\
\text { permanece }\end{array}$ & Tema \\
\hline $\begin{array}{l}\text { Biocomercio } \\
\text { Colombia }\end{array}$ & 26 & 16 & $62 \%$ & $\begin{array}{l}\text { Estrategias sostenibles de comercio (biocomercio) } \\
\text { que fomentan la pertinencia y que van en conso- } \\
\text { nancia con la protección de la biodiversidad y la } \\
\text { preservación de la cultura. }\end{array}$ \\
\hline Convivir & 28 & 14 & $50 \%$ & $\begin{array}{l}\text { En Colombia el desarrollo es factible si se inte- } \\
\text { gran en la gestión ambiental aspectos como el or- } \\
\text { denamiento territorial, la agricultura, la minería, } \\
\text { la construcción de infraestructura, el desarrollo } \\
\text { urbano, teniendo en cuenta lo social y la biodi- } \\
\text { versidad. }\end{array}$ \\
\hline Biomímesis & 25 & 12 & $48 \%$ & $\begin{array}{l}\text { Innovación tecnológica basada en la imitación de } \\
\text { la naturaleza como alternativa para construir mo- } \\
\text { delos sostenibles de desarrollo. }\end{array}$ \\
\hline Disoñadores & 26 & 7 & $27 \%$ & $\begin{array}{l}\text { Alternativas para el desarrollo social y el cuidado } \\
\text { ambiental, basadas en la innovación en el manejo } \\
\text { de la biodiversidad. }\end{array}$ \\
\hline
\end{tabular}

Fuente: elaboración propia.

Colombia tiene, teniendo en cuenta su biodiversidad, con una amplia variedad de alternativas de gestión ambiental. Desde el rescate de la comida tradicional y por tanto de las costumbres culturales explicadas en el artículo "Comer borugo", hasta la capacidad de los habitantes de un territorio de ir más allá de los modos de producción imperantes, e innovar para conseguir una mejor calidad de vida, como así lo señala el artículo "Disoñadores", que refiere los logros de campesinos nariñenses para conservar la naturaleza y su cultura.

Es importante cerrar esta estructura de las relaciones entre los temas de los artículos con la mención de un muy significativo aspecto de la gestión ambiental: la retribución por lo que se toma, expresada explícitamente en el artículo "Pagamentos", y de forma más metaforizada en "El árbol de la abundancia". En la época actual crece la necesidad de pagos por los servicios ambientales o ecosistémicos, que son primordiales para la sostenibilidad; esto conlleva el asumir obligaciones constitucionales, responsabilidades ciudadanas, compromisos ecológicos y políticas fiscales que, de forma clara y legible para todos, permitan reintegrar recursos al ecosistema, en otras palabras, es fundamental una revaloración de los recursos naturales y culturales. En igual medida es conveniente aludir al artículo "Gentileza" donde esa virtud se orienta al nuevo socioecosistema, que nace debido a las interacciones y transiciones mencionadas antes, y que implica oportunidades de vida para todas las especies, y para el bienestar humano más allá de las necesidades básicas, donde haya una reevaluación de las mismas, un trato más justo, y un principio para la paz.

A modo de recapitulación, se reconoce entonces que a nivel global hay tres temas que subyacen en todos los artículos y que son:

- La gestión ambiental de acuerdo con las características de la biodiversidad colombiana.

- Componente social -cultural que interviene en la gestión ambiental.

- Innovación en investigación para conseguir información relevante. Uso de dicha información en la ejecución de prácticas ambientales sostenibles. 
Se reconoce, además, que los artículos poseen una estructura común, con tres partes principales que pueden resumirse así: inicio, desarrollo y conclusión.

- Inicio: Se incluye una referencia real para introducir y contextualizar el tema. Por ejemplo, en el artículo denominado "Pacto ¿agro... ambiental?" (Baptiste, 2013w) la autora empieza con una descripción del sistema de riego Subak, de la isla de Bali, como mecanismo que integra las tradiciones religiosas y culturales de los pobladores, con el modo de producción de comida. En adelante se desprende que toda intervención en dicha práctica y en las que existen a lo ancho del planeta incide en el modo de vida de las comunidades, no solo en cuanto a producción sino en sus costumbres sociales; por tanto, la gestión ambiental se debe ocupar tanto del patrimonio natural como del cultural.

- Desarrollo: Aparecen hechos científicos y datos que soportan la argumentación del tema. En el artículo "Información ambiental 1, 2 y 3" (Baptiste, 2013e) que se ocupa de la inversión en conseguir información ambiental permanente, accesible, relevante, oportuna y oficial, se indican porcentajes que soportan el estado de crisis que vive Colombia, dice la autora: "Se invierte en el Minambiente menos del $1 \%$ del presupuesto nacional [...] en un país que perdió más de $\$ 11$ billones por efecto del fenómeno de La Niña" (Baptiste, 2013e). Lo que genera pobreza $y$, en consecuencia, conflictos sociales.

- Conclusión: Se indican lineamientos de reflexión, como en el artículo "Ayuno y abstinencia" (Baptiste, 2013d) publicado durante la Semana Santa católica de 2013, y cuyo tema es el consumo insostenible de recursos como resultado de la constante búsqueda del placer, promovida por el mercado. Dice el texto: "La reflexión ambiental nos recomienda, al igual que casi todas las escuelas de pensamiento de la antigüedad, místicas o no, ayuno y abstinencia" (Baptiste, 2013d); aquí la autora recurre a las prescripciones de diferentes culturas sobre la sostenibilidad de la vida que deben ser tenidos en cuenta para afrontar la crisis ambiental.

En otros artículos se presentan recomendaciones explícitas de acción que incluyen a la ciudadanía, en especial a los de la urbe como elementos claves de la gestión ambiental; en "Territorios colectivos y biocapacidad" (Baptiste, 2013h); por ejemplo, se habla sobre la necesidad de conformación de instituciones del sector privado que apoyen el mantenimiento de la biocapacidad del territorio.

Pero insisto en la noción de agremiación, ya que la gestión ecológica, aun cuando deba hacerse en todas y cada una de las casas, unidades productivas, industrias o empresas, requiere agregación para tener impacto [...], más allá de la acción agregada, necesitamos de la acción coordinada y persistente de todos los agentes, acción colectiva que sólo es factible con altos niveles de cultura política, confianza e instituciones que hagan la mediación (Baptiste, 2013h).

En igual medida los artículos señalan las responsabilidades a asumir, debido a que tan importante asunto no se ejerce como debería. En el artículo "Nada se toca" (Baptiste, 2013u) se menciona que el rechazo de la población ante el panorama de riesgos ecológicos integrados por, entre otras cosas, rellenos sanitarios rebosantes, actividades petroleras destructoras, deforestación intensiva, agroquímicos mortales:

[...] tenemos que preguntarnos, a fondo, si la suma de estos preceptos constituye una política per se o solo es el idilio, ese sitio poético e imaginario donde no asumimos ninguna responsabilidad y nos arrunchamos, bucólicos, en los sabios brazos de la madre tierra (Baptiste, 2013u). 
A partir de los resultados anteriores se puede enunciar que la principal labor necesaria para afrontar la crisis ambiental es un cambio en la forma como las personas se relacionan con el entorno, es decir, cómo piensan y viven la naturaleza. En la vida cotidiana e incluso en los ámbitos científico, económico e industrial, los seres humanos se consideran un eslabón apartado de los procesos naturales, a los que dirigen su atención para gobernarlos desde la distancia. Eso produce una falla en las relaciones de intercambio, indispensables para el funcionamiento de los ecosistemas, que lleva al desperdicio de recursos y energía.

Baptiste propone con sus artículos un retorno a la apropiada valoración de las acciones y modos de vida que van en consonancia con los procesos naturales, de esta manera recalca que la humanidad es un agente primordial de estos, es una parte del ecosistema, de esta forma la naturaleza es el hogar natural del ser humano. Gracias a la fecundidad y biodiversidad de un territorio ha proliferado la riqueza cultural de los pueblos, lo que indica la existencia de intrincadas relaciones de las que depende la supervivencia humana. El reconocimiento asertivo de esta situación es entonces la base para un cambio real en la forma de relacionarse con el mundo, y es el llamado que invoca reiteradamente la autora. En cada artículo puede observarse la aparición de los fenómenos sociales como determinantes del rumbo de la situación ambiental, es decir que las tradiciones heredadas, la cultura creada, los acontecimientos históricos, las formas de nombrar el mundo, vinculan al ser humano y guían sus decisiones. Tener una perspectiva clara de cómo estas variables intervienen y se conjugan es clave para lo que la autora enuncia como gestión ambiental.

\section{ESTRATEGIAS DISCURSIVAS}

Respecto a las estrategias discursivas identificadas en este nivel semántico, es provechoso observar que la autora utiliza diferentes recursos para transmitir sus ideas. Aparece el uso de referencias locales acerca a la situación de forma contextualizada, así en "La gobernanza de las ficciones territoriales", explica a través de una situación clave, lo que ocurre en Colombia, en donde a pesar de contar con reservas forestales, parques nacionales o páramos definidos:

44 municipios de la cuenca alta del río Bogotá naufragan en las contradicciones creadas por 35 años de ignorar la reserva forestal definida para defender la captación de agua en la región, innumerables áreas de parques nacionales que deberían operar bajo categorías de conservación absoluta están Ilenos de gente a quien ni compensan ni permiten trabajar, y ahora tendremos miles de habitantes de la alta montaña cuyas actividades agropecuarias y modos de vida quedarían severamente restringidas por las buenas intenciones de defender los páramos en la Ley del Plan de Desarrollo (Baptiste, 2013i).

La gobernanza efectiva de todo el territorio nacional requiere el reconocimiento de sus condiciones biológicas y culturales, además de leyes claras y pertinentes de protección de la biodiversidad.

Los datos científicos que presenta se ubican en el texto para reforzar la exhortación a acciones oportunas, como cuando en "Reforma rural integral y servicios ecosistémicos" menciona: "El caso de las 70.000 ha contaminadas con mercurio en el bajo río Cauca por la minería ilegal" (Baptiste, 2013k), para indicar la urgencia de que se protejan los servicios ecosistémicos, los modos de vida y la producción agropecuaria de las comunidades campesinas. El artículo "Travesías, transiciones" está construido de manera que muestra la importancia de conocer el territorio para poder habitarlo, es en el que más se evidencia lo necesario de la contextualización de una situación. Presenta el viaje como forma de entender y proteger mejor el territorio, y crear así una planeación integral y equilibrada del uso del mismo: "Por estos días realizamos una travesía por el río Meta con el ánimo de dar a conocer 
las profundas transformaciones que se están dando en los Llanos Orientales y ampliar la discusión acerca de sus efectos ecológicos y sociales" (Baptiste, 2013n).

Una estrategia usada con frecuencia en los discursos es la metáfora; en el caso de la autora, se emplea este recurso para explicar lo más claro posible una situación. El artículo "Cesar las guerras contra el agua" plantea desde el título una metáfora del conflicto existente entre las personas y la fuente de la vida, lo que es absurdo. En una guerra nadie gana. Los humedales y el ciclo del agua ecuatorial ha sido desde la colonia un reto que se mantiene sin una solución coherente con la realidad del mundo a nivel ecológico y social. El artículo demuestra que mientras se piense en doblegar y someter procesos como el flujo del agua, soporte de la vida, se mantendrá una guerra que arrasa con gentes, territorios, tradiciones y derechos por igual. Manifiesta así que la construcción de una nueva cultura del agua, entendiéndola como motor del desarrollo, implica que se impongan límites no solo a los humedales sino a la insaciabilidad humana; estos ajustes son urgentes, sobre todo ante los fenómenos naturales básicos, que aún hoy siguen desconociéndose -por ejemplo, el ciclo del clima-.

Estos artículos contienen un gran valor más allá de lo informativo, son una fuente de soluciones, debido a que explican las conexiones y relaciones de los principales componentes de una situación de riesgo ambiental; amplían así la perspectiva del lector, formando su criterio ecológico, para eventualmente tomar mejores decisiones ecológicas, que optimizan las condiciones sociales y la calidad de vida.

Esta investigación brinda a la ciudadanía un acceso fácil y oportuno a un aspecto que incide directamente en el bienestar, todo desde la perspectiva crítica que da el ACD. Herramientas digitales como páginas web, cartillas y revistas electrónicas, se presumen como espacios ideales de divulgación de estos hallazgos, ya que ese es el fin último de la investigación: hacer comprensible para todos la problemática ambiental.

\section{COHERENCIA LOCAL}

Retomando los resultados de este apartado de forma general -todos los artículos- se observa que las implicaturas muestran ideas que se reafirman a lo largo del escrito, que se abordan de manera directa, a medida que avanza el artículo. Para las presuposiciones se tomaron algunas de las proposiciones principales del texto, que se relacionaban directamente con el tema, se observa que la proposición resultante confirma lo expuesto por la autora en su escrito.

En los artículos no hay mayor repetición de palabras, y las que se repiten aparecen como un señalamiento que tiene que ver directamente con el tema. De esta forma, la sinonimia es escasa en los artículos, al igual que la comparación; son estrategias poco usadas por la autora, que mantiene un estilo directo y de amplitud de los argumentos.

Se observó que el superordenador está presente en cada uno de los artículos, ya que estos, al tener un tema, cuentan con una noción o tópico que gobierna la lógica del texto. Respecto a las formas de tratamiento, estas son variadas y lexicalizan de forma clara a los actores del artículo.

Se reconoce que en este discurso la construcción de grupos que más se presenta es la dualidad Estado/ciudadanía y rural/urbano, la autora realiza esta distinción para mostrar desde cada perspectiva la situación ecológica y las responsabilidades que cada sector tiene en la gestión ambiental. Aparece con reiteración el adjetivo: expertos, una categorización empleada básicamente para integrar la posición de académicos, investigadores y afines, en la explicación de la problemática ecológica actual, y así pasar a indicar estrategias alternativas para afrontar la crisis desde un enfoque fundamentado en la ciencia y en el conocimiento. 
Al etiquetar a los actores se observa que la autora sitúa a científicos, afrodescendientes, empresas, desplazados, indígenas, campesinos, gobierno, entre otros, en el engranaje social y ecológico que representa la gestión ambiental. Es decir que toda estrategia de intervención y solución debe tenerlos en cuenta como parte fundamental.

La justificación de atribuciones en el discurso global de la autora nace de su visión de la crisis ambiental como un contribuyente en los conflictos sociales actuales, así como de la desvalorización de los recursos -naturales y sociales-, indica en sus artículos las complejas interacciones ecosistémicas, de las que los seres humanos son parte y por tanto participantes de su bienestar o de su decadencia. La ubicación entonces es de una defensora de la gestión ambiental: señala la complejidad de la biodiversidad como la principal fuente de soluciones y de riqueza ecológica, y que genera además una diversidad cultural que es un patrimonio a preservar y revalorar.

\section{CONSIDERACIONES FINALES}

El discurso emitido por la autora posee unas características especiales respecto a su composición lingüística, al vocabulario y al uso de recursos y estrategias discursivas, que debido a la complejidad del tema ecológico contienen diferentes temas y conclusiones, sin embargo se evidencia en este análisis que la intención de Baptiste es una divulgación asertiva y contextualizada de la situación ambiental de Colombia, construye sus textos de forma que pueden ser entendidos por la población, no obstante hace falta contar con conocimientos básicos sobre ecología para comprender los alcances y desenlaces de lo dicho, así mismo poseer una perspectiva histórica, social, económica amplia. A pesar de todo lo anterior, los artículos brindan a aquellas personas que empiezan a acercarse a esta problemática una oportunidad de develar dudas, construir preguntas e indagar, son una propuesta a transformar la manera de relacionarse con el entorno y asumir responsabilidades: una nueva forma de habitar la naturaleza. El señalamiento constante de la autora, de la gran importancia para el manejo de la situación ambiental de todos las construcciones sociales y culturales humanas, es un llamado a una revalorización de los recursos con los que cuenta Colombia, indica que es a partir de la gran diversidad de climas, plantas, animales, lenguas, tradiciones y culturas es posible transformar las condiciones de riesgo ambiental actual y trabajar en el desarrollo de nuevas formas de asumir la naturaleza. Todo esto implica que el ser humano es una parte en el engranaje del ecosistema, que no puede ser tomado como un personaje externo, toda actividad humana tiene un impacto en el conjunto de procesos naturales y estos, a su vez, dictan su modo de vida.

Por último cabe destacar que el desglose que esta investigación plantea sobre el contenido de los artículos de Baptiste contribuye en la comprensión de tema ecológico en Colombia; permite una asimilación más legible de estos; se alcanzaron los objetivos investigativos propuestos, y se espera continuar con la labor de divulgación, que se complementa con los hallazgos del nivel pragmático que se abordarán en futuros escritos.

\section{RECONOCIMIENTOS}

"Reconstrucción analítica de un discurso ecológico" es una investigación concluida, cuyo proyecto se construyó en el semillero Análisis del Lenguaje de la Universidad EAFIT, con la asesoría de la doctora en Humanidades Sonia López. Luego se inscribió en el año 2015 a la Convocatoria de Pequeño Proyecto en la misma universidad, y a partir de su aprobación se financió por el trascurso de un año para que se realizara el trabajo de campo y se culminara el análisis, hecho que tuvo lugar en diciembre de 2015, con la consecución de todo lo esperado. Se presentó el 
informe final a la universidad. El artículo que se presenta a la revista Enunciación es un derivado directo de dicho informe, y resalta un componente específico: el social.

\section{REFERENCIAS BIBLIOGRÁFICAS}

Baptiste, B. (2013a). Punto de partida. Diario La República. Colombia.

Baptiste, B. (2013b). La lora ambiental. Diario La República. Colombia.

Baptiste, B. (2013c). Las economías del páramo. Diario La República. Colombia.

Baptiste, B. (2013d). Ayuno y abstinencia. Diario La República. Colombia.

Baptiste, B. (2013e). Información ambiental 1, 2 y 3. Diario La República. Colombia.

Baptiste, B. (2013f). El lugar de la biodiversidad en la paz. Diario La República. Colombia.

Baptiste, B. (2013g) Desplazamiento ambiental. Diario La República. Colombia.

Baptiste, B. (2013h). Territorios colectivos y biocapacidad. Diario La República. Colombia.

Baptiste, B. (2013i). La gobernanza de las ficciones territoriales. Diario La República. Colombia.

Baptiste, B. (2013j) Innovación ambiental. Diario La República. Colombia.

Baptiste, B. (2013k) Reforma rural integral y servicios ecosistémicos. Diario La República. Colombia.

Baptiste, B. (2013I) OCDE Verde. Diario La República. Colombia.

Baptiste, B. (2013m) Transiciones ecológicas y sociales en Santurbán. Diario La República. Colombia.

Baptiste, B. (2013n). Travesías, transiciones. Diario La República. Colombia.

Baptiste, B. (2013o) Enfermedades ecosistémicas. Diario La República. Colombia.

Baptiste, B. (2013p) Prosperidad con límites. Diario La República. Colombia.

Baptiste, B. (2013q) Ecologías de escala. Diario La República. Colombia.

Baptiste, B. (2013r) Ecología profética. Diario La República. Colombia.
Baptiste, B. (2013s) Empresas de servicios ecosistémicos. Diario La República. Colombia.

Baptiste, B. (2013t) Restaurando. Diario La República. Colombia.

Baptiste, B. (2013u). Nada se toca. Diario La República. Colombia.

Baptiste, B. (2013v) La interfaz ciencia-política. Diario La República. Colombia.

Baptiste, B. (2013w). ¿Pacto agro... ambiental? Diario La República. Colombia.

Baptiste, B. (2013x) Cesar las guerras contra el agua. Diario La República. Colombia.

Baptiste, B. (2013y) Sostenibilidad en desarrollo. Diario La República. Colombia.

Baptiste, B. (2013z) Gentileza. Diario La República. Colombia.

Baptiste, B. (2013aa) Efecto silo. Diario La República. Colombia.

Baptiste, B. (2013bb) Comer borugo. Diario La República. Colombia.

Baptiste, B. (2013cc) Experimentando los límites. Diario La República. Colombia.

Baptiste, B. (2013dd) Plantas del pasado. Diario La República. Colombia.

Baptiste, B. (2013ee) Tierra cansada. Diario La República. Colombia.

Baptiste, B. (2013ff) Ministerio de la fauna. Diario La República. Colombia.

Baptiste, B. (2013gg) Resiliencia. Diario La República. Colombia.

Baptiste, B. (2013hh) Pagamentos. Diario La República. Colombia.

Baptiste, B. (2013ii) IBPES. Diario La República. Colombia.

Baptiste, B. (2014a). Innovación ambiental (2). Diario La República. Colombia.

Baptiste, B. (2014b) El árbol de la abundancia. Diario La República. Colombia.

Baptiste, B. (2014c). Lógicas posconsumo. Diario La República. Colombia.

Baptiste, B. (2014d). La fragilidad ambiental de la cultura. Diario La República. Colombia.

Baptiste, B. (2014e). Biocomercio Colombia. Diario La República. Colombia. 
Baptiste, B. (2014f). Convivir. Diario La República. Colombia.

Baptiste, B. (2014g). Biomímesis. Diario La República. Colombia.

Baptiste, B. (2014h). Disoñadores. Diario La República. Colombia.

Calsamiglia, H. (1999). Las cosas del decir. Barcelona: Editorial Ariel.

Davies, P. (1983). Otros mundos. Barcelona: Biblioteca Científica Salvat.

Londoño, D. (2011) Análisis crítico del discurso y arqueología del saber: dos opciones de estudio de la sociedad. Palabra Clave, 14(1), 101-121.
Martínez, M. (1997). Análisis del discurso. Cohesión, coherencia y estructura semántica de los textos expositivos. Cali: Editorial Universidad del Valle.

Sutton, D. (2008). Fundamentos de ecología. México: Limusa.

Van Dijk, T. (1984). Texto y contexto. Semántica y Pragmática del discurso. Madrid: Ediciones Cátedra.

Van Dijk, T. (1994). Discurso, poder y cognición social. Cátedra UNESCO.

Van Dijk, T. (1998). Estructuras y funciones del discurso. México: Siglo XXI.

Van Dijk, T. (1999). El análisis crítico del discurso. Anthropos, 186, 23-36.

Van Dijk, T. (2008)."Semántica del discurso e ideología". Discurso y Sociedad, 2, 201-261. 